\title{
Spatiotemporal Expression Patterns of Critical Genes Involved in FGF Signaling during Morphogenesis and Odontogenesis of Deciduous Molar in Miniature Pig
}

\section{Wenwen Guo}

Capital Medical University School of Stomatology

\section{Ran Zhang}

Peking University Stomatological Hospital

Lei Hu

Capital Medical University School of Stomatology

Jiangyi Wang

Capital Medical University School of Stomatology

\section{Fu Wang}

Dalian Medical University

Jinsong Wang

Capital Medical University

Chunmei Zhang

Capital Medical University School of Stomatology

\section{Xiaoshan Wu}

Xiangya Hospital Central South University

Songlin Wang ( $\sim$ slwang@ccmu.edu.cn )

Capital Medical University School of Stomatology

\section{Research Article}

Keywords: miniature pig, tooth development, deciduous molar, fibroblast growth factor, dental epithelium, dental mesenchyme

Posted Date: March 5th, 2021

DOl: https://doi.org/10.21203/rs.3.rs-267527/v1

License: (c) (1) This work is licensed under a Creative Commons Attribution 4.0 International License. Read Full License 
Version of Record: A version of this preprint was published at International Journal of Medical Sciences on January 1st, 2022. See the published version at https://doi.org/10.7150/ijms.61798. 


\section{Abstract \\ Background}

The fibroblast growth factor (FGF) pathway plays important role in epithelial-mesenchymal interactions during tooth development. However, how the ligands, receptors, and inhibitors of the FGF pathway get involved into the epithelial-mesenchymal interactions are largely unknown in miniature pigs, which can be used as large animal models for similar tooth anatomy and replacement patterns to humans.

\section{Results}

In this study, we investigated the spatiotemporal expression patterns of critical genes encoding FGF ligands, receptors, and inhibitors in the third deciduous molar of the miniature pig at the cap, early bell, and late bell stages. With the methods of fluorescence in situ hybridization and real time RT-PCR, it was revealed that the expression of $F g f 3, F g f 4, F g f 7$, and $F g f 9$ mRNAs were located mainly in the dental epithelium and underlying mesenchyme at the cap stage. The expression levels of $F g f 3$ and $F g f 7$ in the mesenchyme were upregulated in the early bell stage and then concentrated in the odontoblasts layer in the late bell stage. In contrast, the expression levels of Fgf 4 and $F g f 9$ in the mesenchyme were downregulated from the cap to bell stage. Gene expression analysis also suggested that Fgfr1 and Fgfr3 were the major receptors regulating dental calcification. Furthermore, the inhibitor-coding genes Sprouty 2 and Sprouty 4 were expressed in the epithelium and mesenchyme in the three stages, indicating that elaborate regulation occurred during dental morphogenesis.

\section{Conclusions}

The spatiotemporal expression pattern of FGF signaling provides the foundation for future studies aiming to fine-tune dental morphogenesis and odontogenesis by controlling the interactions between the dental epithelium and mesenchyme, thereby promoting tooth regeneration in large mammals.

\section{Background}

Tooth formation is the result of sequential and reciprocal interactions between the ectoderm-derived epithelium and underlying neural crest-derived mesenchyme [1]. Proteins in the fibroblast growth factor (FGF) family mediate inductive interactions between the dental epithelium (DE) and mesenchyme (DM) during several successive stages of tooth formation [2]. According to sequence similarity and functional properties (such as receptor specificity and binding affinity), FGFs can be subdivided into several subfamilies, including FGF1, FGF4, FGF7, FGF8, FGF9, FGF11, and FGF15 [3-6]. Most FGFs mediate their biological responses as extracellular proteins via binding to and activating cell surface tyrosine kinase FGF receptors, known as fibroblast boom component receptors (FGFR) 1-4 [3]. 
FGF signaling is crucial during development of the tooth epithelium and mesenchyme [7]. Fgf4, Fgf8, and Fgf9 are expressed initially in the dental epithelium and later in the enamel knot, thereby affecting cell proliferation during tooth initiation and subsequent morphogenesis to regulate tooth shape establishment [8-13]. Additionally, Fgf3, Fgf7, and Fgf10 are expressed in the dental mesenchyme of mice [14]. Acting as upstream regulators of FGF signaling, sprouty (Spry) genes were first identified as inhibitors of FGFR-mediated signaling [15-16]. The inhibitor coding genes Spry1, Spry2, and Spry4 are expressed and required for proper molar cusp patterning throughout enamel improvement [12,17-18]. However, it remains unclear whether joint FGF and SPRY expression regulates tooth development by modulating epithelial-mesenchymal interactions in large mammals.

Deciduous molars 3 (DM3) in every jaw quadrant of miniature pig has been used to study tooth development and the cascade initiation of permanent molars [19-21]. This is the first study to characterize the dynamic expression profile of genes related to the FGF signaling pathway in DM3 in miniature pig at the cap, early bell, and late bell (secretory) stages, particularly in relation to cusp patterning and dental calcification. Thus, our study improves the understanding of the interaction between the epithelium and mesenchyme in tooth development, which may be applicable in human tooth regeneration.

\section{Results}

\section{Morphology of DM3 across developmental stages in miniature pig}

In miniature pig, the cap, early bell, and late bell (secretory) developmental stages of the DM3 occurred at embryonic day 40 (E40), E50, and E60, respectively. At E40, the tooth bud of DM3 entered the cap stage, characterized by formation of the primary enamel knot, a central region of epithelium which is the signaling center involved in regulating tooth shape. Further, the epithelial bud that underwent specific folding and was composed of inner enamel epithelium (IEE), outer enamel epithelium (OEE), stellate reticulum (SR), and intermediate cell layer (Fig. 1a-b). At this stage, the cervical loop, formed by the IEE and OEE together with the SR, was intumescent (Fig. 1C). The typical feature of E50 was formation of secondary enamel knots and beginning of crown morphology and differentiation with the bell shape (Fig. 1d-e). Moreover, the cervical loop elongated and extended towards the future root (Fig. 1f). Finally, at E60, DM3 transited to the later bell stage and exhibited thin DE and bulky DM; additionally, cells at the tips of the IEE and DM differentiated into ameloblasts and began to from pre-enamel prisms and preodontoblasts regions (Fig. 1g-h). Further, the cervical loop, formed by the IEE and OEE with minor contribution of the SR, elongated towards the direction of the future root (Fig. 1i). Thus, the study of DM3 development at stages $\mathrm{E} 40$ to $\mathrm{E} 60$ could reveal information on the processes of cusp shaping, crown calcification, and root cytodifferentiation in miniature pig.

\section{Dynamic expression of genes encoding FGF ligands and inhibitors during morphogenesis of DM3}

Fgf3, 4, 7 and 9 mRNAs are central components of the typical FGF signaling pathway, whereas Spry2 and Spry 4 are key inhibitors [12]. Therefore, we examined these genes expression patterns of in both the 
epithelium and mesenchyme of DM3 at the E40, E50, and E60 stages by fluorescence in situ hybridization (FISH) and immunofluorescence (IF). Cytokeratin 14 protein was used to mark the margin of the tooth epithelium. Fgf3 mRNA mainly presented in the IEE at E40 (Fig. 2a). However, it was expressed mainly in the dental mesenchyme, specifically in the pre-odontoblasts and odontoblast cell layer at E50 and E60 (Fig. 2b-c). Fgf3 mRNA was present in the cervical loop in the three stages (Supplementary Fig. 1a-c). Fgf4 mRNA was expressed in the enamel knot and dental mesenchyme at E40 (Fig. 2d) and E50 (Fig. 2e). Interestingly, Fgf4 mRNA was expressed weakly at E60 (Fig. 2f). In addition, Fgf4 mRNA was present only in the IEE of the cervical loop at E40 and E50 (Supplementary Fig. 1d-f).

At the cap stage, Fgf7 mRNA was detected in both the DE and DM, with much stronger expression observed at E50 (Fig. 2g-h). However, the expression of Fgf7 was restricted in odontoblasts at E60 (Fig. 2i). Fgf9 mRNA was mainly expressed in the IEE and dental mesenchyme at E40. However, the expression level was greatly reduced at E50 and E60 (Fig. 2j-I). Additionally, Fgf7mRNA was present in the cervical loop in the three stages (Supplementary Fig. 1g-i). Fgf9 mRNA was present in the IEE of the cervical loop at E40 and E50 (Supplementary Fig. 1j-I). Thus, Fgf3 and Fgf7 mRNAs were upregulated in the mesenchyme from E40 to E50, and subsequently found to be concentrated in the odontoblast layer at E60. Conversely, Fgf4 and Fgf9 mRNAs in the mesenchyme were decreased from E40 to E60.

Next, we studied the expression patterns of the inhibitors of FGF signaling. The results showed that Spry2 was present in the DE at E40 (Fig. 3a). At E50, Spry2 mRNA was highly expressed in the IEE and underlying DM (Fig. 3b). Interestingly, Spry2 was mainly expressed in DM at E60 (Fig. 3c). However, Spry4 mRNA was present in both the DE and DM at E40 (Fig. 3d). Spry4 mRNA was mainly expressed in the DM at E50 and E60 (Fig. 3e-f). As a result, inhibitors of Spry2 and 4 were downregulated in the DE but upregulated in the DM during odontogenesis. Additionally, Spry2 and Spry4 mRNAs were present in the cervical loop in the three stage (Supplementary Fig. 2).

\section{Distribution of FGF ligand proteins during morphogenesis of DM3}

Next, to confirm the results of FISH, we examined the distribution of several FGF proteins at the three different stages of DM3 development by immunofluorescence. We found that FGF3 protein was mainly expressed in the DE at E40, in the both DE and DM at E50, and in the ameloblast and odontoblast layer at E60 (Fig. 4a-C). FGF4 protein was expressed in the SR of DE and DM at E40, but largely reduced at E50 and E60 (Fig. 4d-f). We found that the expression level of FGF7 protein was gradually increased in the DM from E40 to E60 (Fig. 4g-i). The expression level of FGF9 protein was gradually decreased both in the DE and DM from E40 to E60 (Fig. 4j-I). The distinct patterns of FGF ligand proteins indicate the elaborate role of FGF signaling during dental morphogenesis and odontogenesis.

\section{Dynamic expression of genes encoding FGF receptors during morphogenesis of DM3}

FGF ligands bind FGF receptors on the surface of target cells to transfer signals to the nucleus. We performed FISH to examine the expression of Fgfr1, Fgfr2, and Fgfr3 mRNAs in DM3 at the three developmental stages. Fgfr1 mRNA was mainly expressed in the DM at E40, and both in the DE and DM 
at E50 (Fig. 5a-b). However, the expression levels were greatly reduced at E60 (Fig. 5c) except for in the odontoblast cell layer and cervical loop (Supplementary Fig. 3a-c). A similar but weaker expression pattern was observed for Fgfr2 mRNA at the three stages (Fig. 5d-f). Finally, Fgfr3 was mainly present in the IEE and underlying DM at E40 (Fig. 5g). Interestingly, Fgfr3 expression was restricted to the DM from E50 to E60 (Fig. 5h-i), and only limited to secretory pre-odontoblasts at E60.

Additionally, Fgfr2 and Fgfr3 transcripts were present in the cervical loop from E40 and E50 (Supplementary Fig. 3d-i). As a result, the mRNA expression levels of FGF receptors were downregulated in the $\mathrm{DE}$, upregulated in the $\mathrm{DM}$, and restricted in the odontoblasts during odontogenesis.

\section{Quantitative gene expression dynamics related to the FGF pathway and its inhibitors of DM3}

To further investigate the expression dynamics of FGF-related genes and inhibitors, we quantified their mRNA levels by real time RT-PCR and analyzed the expression differences of each gene in both the DE and DM across the three stages. Tooth germs of DM3 were harvested and the epithelium was separated from the mesenchyme by dispase II treatment before reverse transcription [22]. The results of real time RT-PCR confirmed that Fgf3 and Fgf7 showed relatively high expression levels in the DM with respect to the DE from E40 and E50 (Fig. 6a, c). However, Fgf4 and Fgf9 mRNAs were remarkably more present in the DE than in the DM from E40 and E50 (Fig. 6b, d). These results were consistent with those of FISH and suggested that these genes were primarily involved in early epithelium growth and formation of the enamel knot. Fgfr1, Fgfr2, Fgfr3 mRNAs were more abundant in the DM than in the DE at the three stages; Fgfr1 and Fgfr3 were highly expressed in the DM at E60 (Fig. 6e-g).

Similar to the expression detected by FISH, the FGF inhibitor Spry2 showed slightly higher expression in the DE at E40 and markedly higher expression in the DM at E50 and E60 (Fig. 6h). In contrast, Spry4 mRNA was more notably accumulated in the DM than in the DE at all three stages (Fig. 6i), with relatively higher expression at E60.

\section{Discussion}

The roles of FGF signal in tooth development of miniature pig are not well-understood. In this study, we examined the dynamic expression of representative FGF ligands and receptors in addition to inhibitors of DM3 at the E40, E50, and E60. We found that the expression levels of Fgf3, Fgf7, Spry2, Spry4, Fgfr1, Fgfr2, and Fgfr3 were increased in the mesenchyme from E40 to E50, and then concentrated in the odontoblast layer at E60. In contrast, the expression levels of $F g f 4$ and $F g f 9$ in the mesenchyme were decreased from E40 to E60. Most molecules were expressed in the epithelium at the cap stage but were gradually reduced when the tooth bud entered the late bell stage. The related gene pattern diagram was shown in detail (Fig. 7). Overall, the FGF signaling pathway and sprouty genes may communicate between the epithelium and mesenchyme to maintain the tooth morphology and mineralization.

We compared the results with those of the previous studies. We found both the similarities and differences among the species of mouse [23], pig and human [24] (Table 1). As an important factor, FGF3 has been found to play a significant role in cell proliferation and morphogenesis of tooth development 
[12]. In human, Fgf3 was expressed mainly in the mesenchyme, particularly in the pre-odontoblasts and odontoblast cell layer at the later stage [24]. These findings were similar with ours. Additionally, Fgf7 expression was detected both in the DE and in the DM at the cap stage of the human tooth [24]. However, Fgf7 may not be involved in mouse tooth development [11]. In our study, the expression of Fgf7 was remarkable in the DM at the early bell stage but restricted in the odontoblast layer at the secretory stage in the DM3. Fgf4 and Fgf9 mRNAs are indispensable for the DE in mice tooth growth [2]. In our study, Fgf4 and Fgf9 were broadly expressed not only in the DE, but also in the DM from the cap to the early bell stage of DM3, although the functional role in the DM has not been elucidated. According to our results, the ligands of $\mathrm{Fgf3}, 4,7$, and 9 may play critical roles in the cusp patterning, whereas only Fgf3 and Fgf7 may play fundamental role in the cell differentiation of odontoblasts.

Table 1

Comparison of the expression of FGF signaling pathway and inhibitors between mouse, pig and human

\begin{tabular}{|c|c|c|c|c|}
\hline \multirow[t]{2}{*}{ stage } & \multirow[t]{2}{*}{ tissues/cells } & \multicolumn{3}{|l|}{ species } \\
\hline & & Mouse [23] & Pig & Human [24] \\
\hline \multirow[t]{2}{*}{$\begin{array}{l}\text { cap } \\
\text { stage }\end{array}$} & $\begin{array}{l}\text { Enamel } \\
\text { knot/Dental } \\
\text { epithelium }\end{array}$ & $\begin{array}{l}\text { Fgf3, Fgf4, Fgf9, Fgf15, } \\
\text { Fgf20, Fgf16, Fg17, } \\
\text { Fgfr1囚b, Fgfr2邓b, Fgfr2『c, } \\
\text { Spry2, Spry4 }\end{array}$ & $\begin{array}{l}\text { Fgf3, Fgf4, Fgf7, } \\
\text { Fgf9, Fgfr1, } \\
\text { Fgfr2, Fgfr3, } \\
\text { Spry2, Spry4 }\end{array}$ & $\begin{array}{l}\text { Fgf3, Fgf4, Fgf7, } \\
\text { Fgf8, Fgf9, } \\
\text { Fgf10, Fgfr1, } \\
\text { FGFR2, Fgfr3 }\end{array}$ \\
\hline & dental papilla & $\begin{array}{l}\text { Fgf3, Fgf10, Fgf16, Fgf17, } \\
\text { Fgf18, Fgfr1凶c, Fgfr2囚c, } \\
\text { Spry4 }\end{array}$ & $\begin{array}{l}\text { Fgf3, Fgf4, Fgf7, } \\
\text { Fgf9, Fgfr1, } \\
\text { Fgfr2, Fgfr3, } \\
\text { Spry2, Spry4 }\end{array}$ & $\begin{array}{l}\text { Fgf3, Fgf4, Fgf7, } \\
\text { Fgf8, Fgf9, } \\
\text { Fgf10, Fgfr1, } \\
\text { Fgfr2, Fgfr3 }\end{array}$ \\
\hline \multirow[t]{2}{*}{$\begin{array}{l}\text { bell } \\
\text { stage }\end{array}$} & $\begin{array}{l}\text { Enamel knot/ } \\
\text { Dental } \\
\text { epithelium }\end{array}$ & 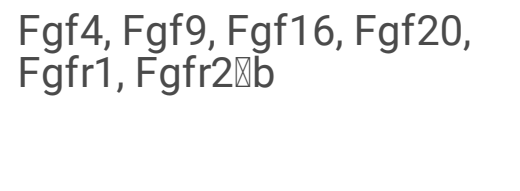 & $\begin{array}{l}\text { Fgf3, Fgf4, Fgf7, } \\
\text { Fgf9, Fgfr1, } \\
\text { Fgfr2, Spry2 }\end{array}$ & $\begin{array}{l}\text { Fgf3, Fgf4, Fgf7, } \\
\text { Fgf8, Fgf9, } \\
\text { Fgf10, Fgfr1, } \\
\text { Fgfr3 }\end{array}$ \\
\hline & dental papilla & $\begin{array}{l}\text { Fgf3, Fgf10, Fgf15, } \\
\text { Fgfrilllb, Fgfr1lllc, Fgfr2Illc }\end{array}$ & $\begin{array}{l}\text { Fgf3, Fgf4, Fgf7, } \\
\text { Fgfri, Fgfr2, } \\
\text { Fgfr3 Spry2, } \\
\text { Spry4 }\end{array}$ & $\begin{array}{l}\text { Fgf7, Fgf8, Fgf9, } \\
\text { Fgfr1 }\end{array}$ \\
\hline \multirow[t]{4}{*}{$\begin{array}{l}\text { secretory } \\
\text { stage }\end{array}$} & $\begin{array}{l}\text { ameloblast/ } \\
\text { Dental } \\
\text { epithelium }\end{array}$ & $\begin{array}{l}\text { Fgf2, Fgf4, Fgf9, Fgf9, } \\
\text { Fgf16, Fgfr1, Fgfr2lllb }\end{array}$ & $\begin{array}{l}\text { Fgf3, Fgf7, Fgfr1, } \\
\text { Spry2 }\end{array}$ & \\
\hline & $\begin{array}{l}\text { odontoblast/pre- } \\
\text { odontoblast }\end{array}$ & Fgfr1IIIb, Fgfr1IIIc & $\begin{array}{l}\text { Fgf3, Fgf7, } \\
\text { Spry2, Fgfr1, } \\
\text { Fgfr2, Fgfr3, }\end{array}$ & \\
\hline & & & Spry2, Spry4 & \\
\hline & dental papilla & Fgf15 & $\begin{array}{l}\text { Fgf3, Fgfr1, } \\
\text { Fgfr2, Spry2, } \\
\text { Spry4 }\end{array}$ & \\
\hline
\end{tabular}


FGF ligands bind to its receptors and activate intracellular signal transduction pathways by inducing receptor phosphorylation during tooth growth. In humans, FGF7 binds specifically to FGFR2, whereas FGF3 and FGF10 bind to both FGFR1 and FGFR2 [25-26]. With the transgenic mice, Fgfr1 null allele heterozygosity reduced the frequency of supernumerary tooth formation to $60 \%$, and $F g f r 2$ null allele heterozygosity reduced it to $0 \%$ [27-28]. In our study, Fgfr1, Fgfr2, and Fgfr3 were mainly expressed in DM cells at the three stages of DM3. However, these receptors related to gene expression and function networks require further analysis in miniature pigs.

Acting as inhibitors of FGF signaling, different sprouty genes cooperate to ensure the correct morphology and size of developing teeth. In mice, Spry2 expression can be observed throughout the epithelium, whereas Spry 4 mRNA accumulates exclusively in the dental mesenchyme to prevent the development of supernumerary teeth [12]. In the mouse incisor, which is a continuously growing tooth, Spry2 and Spry4 restrict the differentiation of enamel-secreting ameloblasts to the labial side, thereby allowing asymmetric enamel deposition [29]. In this study, we found that, unlike in mice [12], Spry2 was expressed in the DE firstly, then expressed in DE and DM but expressed mostly in the DM from the cap to the later bell stage, and Spry2 may be transferred from the epithelium to the dental mesenchyme to regulate tooth development. Spry4 expression was enhanced in the DM from the cap stage to the secretory stage. As the inhibitors of FGF signaling, the Sprys may lead to a potential gradient of FGF signaling to regulate tooth morphogenesis and odontogenesis precisely.

The interaction among several FGF family members in the IEE and dental mesenchyme regulates the differentiation of dentin and subsequent dentin formation [30]. Particularly, FGF4 and FGF9 expressed in epithelial cells are thought to maintain FGF3 expression in the dental mesenchyme to further regulate epithelial cell proliferation and morphogenesis [31]. Specifically, FGF3 stimulates the proliferation of inner enamel epithelial cells, whereas FGF7 stimulates the proliferation of outer enamel epithelial cells [31-32]. In contrast, loss of Spry2 function in the epithelium leads to the formation of diastema teeth buds due to the expression of Fgf3 in the mesenchyme, which was sufficient to control expression of Shh and perhaps also Fgf4. The normal function of Spry 4 in the mesenchyme is to prevent the perception of any epithelial FGF signals, including FGF4 and FGF9 produced in the adjacent M1 tooth germ, to induce or maintain FGF3 expression [12]. Based on the adverse localization and relevant pattern of Fgf4 and Spry4 in the DE as well as Fgf3 and Spry2 in the DM of DM3, we predicted the existence of a feedback mechanism that precisely regulates FGF signaling to mediate the interaction between the epithelium and mesenchyme during tooth development, which is needed to further exploration (Supplementary Fig. 4).

\section{Conclusions}

In this study, we revealed the spatiotemporal distribution and the potential gradient of FGF and Sprouty molecules during tooth odontogenesis in large mammals. Modulation of the epithelium-mesenchyme interactions by fine-tuning the FGF pathway may promote the cusp patterning and dental calcification in the future studies. 


\section{Methods}

\section{Animals}

Pregnant miniature pigs were obtained from the Animal Science Institute of Chinese Agriculture University. The gestation age was calculated from the day of insemination. Pregnancy was verified by Btype ultrasonography. All procedures were approved by the Animal Care Use Committee of Capital Medical University (Beijing, China) (Permit Number: AEEI-T-212). Pregnant miniature pigs were anesthetized and sacrificed as previously described [33]. The DM3 and mandible were harvested at embryonic days 40 (E40), E50, and E60, which corresponded to the cap, early, and late bell (secretory) stages.

\section{Tissue preparation for HE and immunofluorescence}

Mandibular samples were fixed with paraformaldehyde, $4 \%$ in phosphate-buffered saline (PBS), at $4^{\circ} \mathrm{C}$ overnight. The samples were rinsed with PBS twice before being decalcified with 10\% EDTA-PBS for 3-14 days according to the degree of calcification. The samples were dehydrated in an ethanol series and embedded in paraffin. Paraffin-embedded samples were sectioned at $7 \mu \mathrm{m}$ thickness for later staining. Sections were stained with hematoxylin and eosin and observed by whole slide image (Pannoramic scan, 3DHistech, Budapest, Hungary). The images were processed with Case Viewer software (3DHistech, Budapest, Hungary). Immunofluorescence (IF) was performed as described previously [34]. Briefly, deparaffinized sections were microwaved for 3 min in citrate buffer ( $\mathrm{pH}$ 6.0). Then the slides were reheated with adding additional buffer, which processes were repeated for three times for antigen retrieval. When the slides were cooled down to room temperature, non-specific antigens were blocked with $10 \%$ normal goat serum (C-0005, Bioss, Beijing, China) for $1 \mathrm{~h}$. The sections were incubated with primary antibodies at $4^{\circ} \mathrm{C}$ overnight. The primary antibodies used in this research were as follows: rabbit antiFGF3 antibody (bs-1255R, Bioss, Beijing, China), rabbit anti-FGF4 antibody (bs-1256R, Bioss, Beijing, China), rabbit anti-KGF antibody (bs-0734R, Bioss, Beijing, China), rabbit anti-FGF8 antibody (bs-0735R, Bioss, Beijing, China), and rabbit anti-FGF9 antibody (bs-5906R, Bioss, Beijing, China). Donkey anti-Rabbit IgG $(\mathrm{H}+\mathrm{L})$, Alexa Fluor 594 (A-21207; Thermo, CA, USA) as secondary antibody was subsequently applied at $37^{\circ} \mathrm{C}$ for $1 \mathrm{~h}$. The nuclei were stained with DAPI (F6057, Sigma, St. Louis, MO, USA). Finally, the sections were observed using confocal microscopy (TCS SP8, Leica, Wetzlar, Germany). Objective lenses were used $20 x$ and $40 \times$, N.A. 0.75. The images were processed with LAS AF software.

\section{Fluorescence in situ hybridization}

Fluorescence in situ hybridization (FISH) staining was performed using tooth tissues fixed in 4\% DEPCtreated paraformaldehyde/PBS, embedded in paraffin, and sectioned at $7 \mu \mathrm{m}$. The procedure for nonradioactive in situ hybridization was described previously [35]. Briefly, total RNA was extracted from DM3 tooth germs at E40-E60. Sequences of the degenerate primers for Fgf3, 4, 7, 9, Fgfr1, 2, 3, and Spry2, 4 were listed in Supplementary Table 1. After reverse transcriptase-polymerase chain reaction, the correctsized bands were extracted from agarose gels and their DNA sequences were determined. Digoxigenin- 
(DIG)-labeled RNA probes were synthesized with DIG-UTP and T7 RNA polymerase (10881767001; Roche, Basel, Switzerland) and DIG RNA labeling mix solution (11277073910; Roche, Basel, Switzerland). Sections were prepared using standard procedures and then incubated in horseradish peroxidaseconjugated, polyclonal sheep anti-digoxigenin antibody (90420, Merck Millipore) at $37^{\circ} \mathrm{C}$ for $4 \mathrm{~h}$. Immunoreactive cells were visualized with TSA Plus Fluorescence Kits (NEL741001KT, PerkinElmer, Massachusetts, USA). Paraffin sections were processed for mucin 2 staining with rabbit anti-Cytokeratin14 (ab119695, Abcam, Cambridge, England). Donkey anti-Rat IgG $(\mathrm{H}+\mathrm{L})$, Alexa Fluor 488 (A-21208; Thermo, CA, USA) as secondary antibody was subsequently applied at $37^{\circ} \mathrm{C}$ for $1 \mathrm{~h}$. Meanwhile. Slides were counterstained with DAPI, mounted and imaged on a Leica TCS SP8 confocal microscope. Objective lenses were used $20 x$ and 40x, N.A. 0.75. The images were processed with LAS AF software.

\section{Real-time RT-PCR}

DM3 tooth germs at E40, E50, and E60 were harvested and the dental epithelium was separated from the

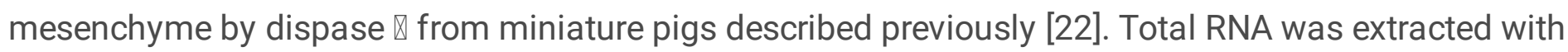
RNeasy Mini Kit (74106, Qiagen, Hilden, Germany) according to its supplied method. Reverse transcription was performed using the SuperScript III First-Strand Synthesis System (18080051; Thermo Fisher Scientific). Real-time RT-PCR was conducted in triplicate using SYBR Green PCR Master Mix (A25742; Applied Biosystems, Foster City, CA, USA) on a CFX96 Touch Real-time RT-PCR Detection System (Bio-Rad Laboratories, Hercules, CA, USA), after which melting curve analysis was performed. Expression level of each gene was normalized to that of Gapdh. The relative expression of each gene was determined using the $2^{-\triangle \triangle C T}$ method. Forward and reverse primers for Fgf3, 4, 7, 9, Fgfr1, 2, 3, and Spry2, 4 and Gapdh were listed in Supplementary Table 2.

\section{Statistical analysis}

Statistical analysis was performed using GraphPad Prism 8.3 software. One-way analysis of variance and Newman-Keuls multiple comparison test were used to calculate statistical significance. Differences were considered to be statistically significant if $p<0.05(*), p<0.01(* *)$, or $p<0.001(\star \star *)$.

\section{Abbreviations}

FGF: fibroblast growth factor; Fgfr: FGF receptors; Spry: Sprouty; DM3: the third deciduous molar; E40: embryonic day 40; E50: embryonic day 50; E60:embryonic day 60; DE: dental epithelium; DM: dental mesenchyme; IEE: inner enamel epithelium; OEE: outer enamel epithelium; SR: stellate reticulum; O: odontoblasts; E: Enamel; pD: pre-dentin; FISH: fluorescence in situ hybridization; IF: immunofluorescence; Gapdh: glyceraldehyde-3-phosphate dehy-drogenase; RT-PCR: Reverse Transcription-quantitative Polymerase Chain Reaction; K14: Cytokeratin 14

\section{Declarations}

Ethics approval and consent to participate 
All procedures were approved by the Animal Care Use Committee of Capital Medical University (Beijing, China) (Permit Number: AEEI-2018-212).

\section{Consent for publication}

Not applicable.

\section{Availability of data and material}

All data generated or analyzed during this study are included in this published article and its supplementary information files.

\section{Competing interests}

Authors declare no conflict of interests.

\section{Funding}

This work was supported by grants from the Chinese Research Unit of Tooth Development and Regeneration, CAMSI Innovation Fund for Medical Sciences, No. 2019-12M-5-031; National Natural Science Foundation of China (91649124 to S.W.); Beijing Municipal Science \& Technology Commission No. Z181100001718208; Beijing Municipal Education Commission No. 119207020201; and Beijing Hospitals Authority of Hospitals' Mission Plan, code『SML20151401; and Beijing Municipality Government grants (Beijing Scholar Program- PXM2018_014226_000021;

PXM2018_193312_000006_0028S643_FCG; PXM2019_014226_000011; PXM2020_014226_000005; Z181100001718208).

\section{Authors' contributions}

SLW and SXW contributed equally to this work. WWG performed the majority of experiments, with additional contribution from RZ, FW, JYW, and JSW. WWG, LH and CMZ analyzed the data and prepared the first draft of the manuscript, which was further edited by SLW and SXW. RZ kindly provided the technical support of FISH. The author(s) read and approved the final manuscript.

\section{Acknowledgements}

We acknowledge the excellent technical assistance from the Transgenic Facility, Animal Facility, and Imaging Core at the University of Capital Medical and Capital Medical University School of Stomatology.

\section{References}

1. Zhang YD, Chen Z, Song YQ, Liu C, Chen YP: Making a tooth: growth factors, transcription factors, and stem cells. Cell Res 2005, 15(5):301-316. 
2. Nie X, Luukko K, Kettunen P: FGF signalling in craniofacial development and developmental disorders. Oral Dis 2006, 12(2):102-111.

3. Itoh N, Ornitz DM: Evolution of the Fgf and Fgfr gene families. Trends Genet 2004, 20(11):563-569.

4. Itoh N, Ornitz DM: Fibroblast growth factors: from molecular evolution to roles in development, metabolism and disease. J Biochem 2011, 149(2):121-130.

5. Popovici C, Roubin R, Coulier F, Birnbaum D: An evolutionary history of the FGF superfamily. Bioessays 2005, 27(8):849-857.

6. Maddaluno L, Urwyler C, Werner S: Fibroblast growth factors: key players in regeneration and tissue repair. Development 2017, 144(22):4047-4060.

7. Liu C, Gu S, Sun C, Ye W, Song Z, Zhang Y, Chen Y: FGF signaling sustains the odontogenic fate of dental mesenchyme by suppressing beta-catenin signaling. Development 2013, 140(21):4375-4385.

8. Jernvall J, Kettunen P, Karavanova I, Martin LB, Thesleff I: Evidence for the role of the enamel knot as a control center in mammalian tooth cusp formation: non-dividing cells express growth stimulating Fgf-4 gene. Int J Dev Biol 1994, 38(3):463-469.

9. Vaahtokari A, Aberg T, Jernvall J, Keranen S, Thesleff I: The enamel knot as a signaling center in the developing mouse tooth. Mech Dev 1996, 54(1):39-43.

10. Kettunen P, Thesleff I: Expression and function of FGFs-4, -8 , and -9 suggest functional redundancy and repetitive use as epithelial signals during tooth morphogenesis. Dev Dyn 1998, 211(3):256-268.

11. Kettunen $P$, Laurikkala J, Itaranta P, Vainio $S$, Itoh N, Thesleff I: Associations of FGF-3 and FGF-10 with signaling networks regulating tooth morphogenesis. Dev Dyn 2000, 219(3):322-332.

12. Klein OD, Minowada G, Peterkova R, Kangas A, Yu BD, Lesot H, Peterka M, Jernvall J, Martin GR: Sprouty genes control diastema tooth development via bidirectional antagonism of epithelialmesenchymal FGF signaling. Dev Cell 2006, 11(2):181-190.

13. Tapaltsyan V, Charles C, Hu J, Mindell D, Ahituv N, Wilson GM, Black BL, Viriot L, Klein OD: Identification of novel Fgf enhancers and their role in dental evolution. Evol Dev 2016, 18(1):31-40.

14. Charles C, Lazzari V, Tafforeau P, Schimmang T, Tekin M, Klein O, Viriot L: Modulation of Fgf3 dosage in mouse and men mirrors evolution of mammalian dentition. Proc Natl Acad Sci U S A 2009, 106(52):22364-22368.

15. Hanafusa H, Torii S, Yasunaga T, Nishida E: Sprouty1 and Sprouty2 provide a control mechanism for the Ras/MAPK signalling pathway. Nat Cell Bio/ 2002, 4(11):850-858.

16. Mason JM, Morrison DJ, Basson MA, Licht JD: Sprouty proteins: multifaceted negative-feedback regulators of receptor tyrosine kinase signaling. Trends Cell Bio/ 2006, 16(1):45-54.

17. Marangoni P, Charles C, Tafforeau P, Laugel-Haushalter V, Joo A, Bloch-Zupan A, Klein OD, Viriot L: Phenotypic and evolutionary implications of modulating the ERK-MAPK cascade using the dentition as a model. Sci Rep 2015, 5:11658.

18. Percival CJ, Marangoni P, Tapaltsyan V, Klein O, Hallgrimsson B: The Interaction of Genetic Background and Mutational Effects in Regulation of Mouse Craniofacial Shape. G3 (Bethesda) 2017, 
7(5):1439-1450.

19. Wang S, Liu Y, Fang D, Shi S: The miniature pig: a useful large animal model for dental and orofacial research. Oral Dis 2007, 13(6):530-537.

20. Xu J, Zheng Z, Fang D, Gao R, Liu Y, Fan ZP, Zhang CM, Wang SL: Early-stage pathogenic sequence of jaw osteoradionecrosis in vivo. J Dent Res 2012, 91(7):702-708.

21. Wang F, Li Y, Wu X, Yang M, Cong W, Fan Z, Wang J, Zhang C, Du J, Wang S: Transcriptome analysis of coding and long non-coding RNAs highlights the regulatory network of cascade initiation of permanent molars in miniature pigs. BMC Genomics 2017, 18(1):148.

22. Wang F, Xiao J, Cong W, Li A, Song T, Wei F, Xu J, Zhang C, Fan Z, Wang S: Morphology and chronology of diphyodont dentition in miniature pigs, Sus Scrofa. Oral Dis 2014, 20(4):367-379.

23. Du W, Du W, Yu H: The Role of Fibroblast Growth Factors in Tooth Development and Incisor Renewal. Stem Cells Int 2018, 2018:7549160.

24. Huang F, Hu X, Fang C, Liu H, Lin C, Zhang Y, Hu X: Expression profile of critical genes involved in FGF signaling pathway in the developing human primary dentition. Histochem Cell Biol 2015, 144(5):457-469.

25. Miki T, Bottaro DP, Fleming TP, Smith CL, Burgess WH, Chan AM, Aaronson SA: Determination of ligand-binding specificity by alternative splicing: two distinct growth factor receptors encoded by a single gene. Proc Natl Acad Sci U S A 1992, 89(1):246-250.

26. Hoffman MP, Kidder BL, Steinberg ZL, Lakhani S, Ho S, Kleinman HK, Larsen M: Gene expression profiles of mouse submandibular gland development: FGFR1 regulates branching morphogenesis in vitro through BMP- and FGF-dependent mechanisms. Development 2002, 129(24):5767-5778.

27. Trokovic N, Trokovic R, Mai P, Partanen J: Fgfr1 regulates patterning of the pharyngeal region. Genes Dev 2003, 17(1):141-153.

28. Yu K, Xu J, Liu Z, Sosic D, Shao J, Olson EN, Towler DA, Ornitz DM: Conditional inactivation of FGF receptor 2 reveals an essential role for FGF signaling in the regulation of osteoblast function and bone growth. Development 2003, 130(13):3063-3074.

29. Klein OD, Lyons DB, Balooch G, Marshall GW, Basson MA, Peterka M, Boran T, Peterkova R, Martin GR: An FGF signaling loop sustains the generation of differentiated progeny from stem cells in mouse incisors. Development 2008, 135(2):377-385.

30. Koussoulakou DS, Margaritis LH, Koussoulakos SL: A curriculum vitae of teeth: evolution, generation, regeneration. Int J Biol Sci 2009, 5(3):226-243.

31. Harada H, Toyono T, Toyoshima K, Yamasaki M, Itoh N, Kato S, Sekine K, Ohuchi H: FGF10 maintains stem cell compartment in developing mouse incisors. Development 2002, 129(6):1533-1541.

32. Harada $\mathrm{H}$, Ohshima $\mathrm{H}$ : New perspectives on tooth development and the dental stem cell niche. Arch Histol Cytol 2004, 67(1):1-11.

33. Li A, Song T, Wang F, Liu D, Fan Z, Zhang C, He J, Wang S: MicroRNAome and expression profile of developing tooth germ in miniature pigs. PLoS One 2012, 7(12):e52256. 
34. Wu X, Hu J, Li G, Li Y, Li Y, Zhang J, Wang F, Li A, Hu L, Fan Z et al: Biomechanical stress regulates mammalian tooth replacement via the integrin beta1-RUNX2-Wnt pathway. EMBO J 2020, 39(3):e102374.

35. Li XB, Yang G, Zhu L, Tang YL, Zhang C, Ju Z, Yang X, Teng Y: Gastric Lgr5(+) stem cells are the cellular origin of invasive intestinal-type gastric cancer in mice. Cell Res 2016, 26(7):838-849.

\section{Figures}
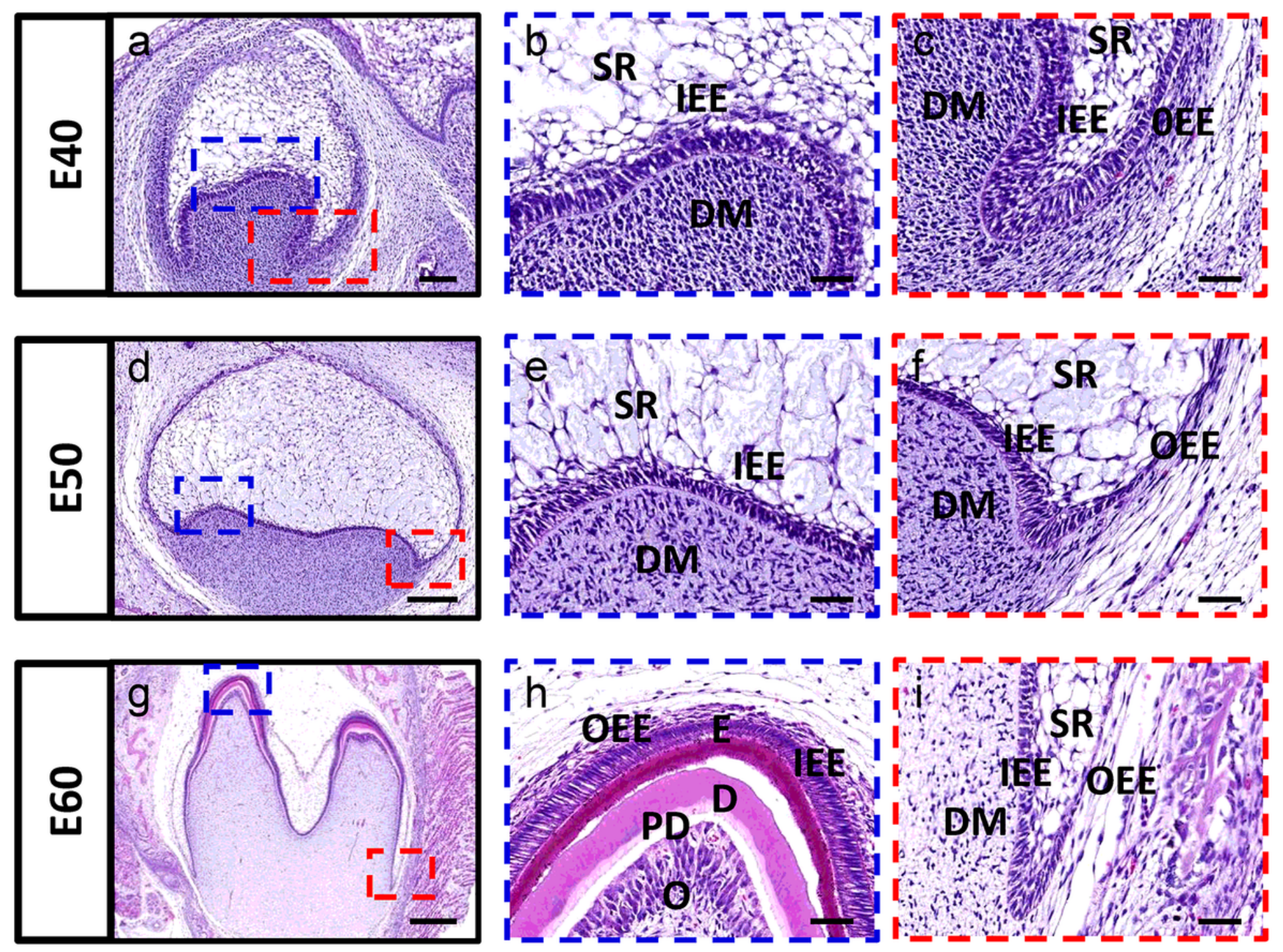

Figure 1

Morphology of DM3 across developmental stages in miniature pig. (a-i) H\&E staining of different stages during tooth germ morphogenesis; Boxed regions in a, d, g were magnified in b-c, e-f, h-i. (a-c) Tooth germ developed into the cap stage at embryonic day 40 (E40). The epithelium folded into the bud and the inner enamel epithelium (IEE) and outer enamel epithelium (OEE) were separated. The primary enamel knot 
began forming at the tip of IEE. The cervical loop was formed by the IEE and OEE joined with stellate reticulum (SR). (d-f) At E50, the DM3 reached the early bell stage and secondary enamel knots began to form. The outer layer of dental mesenchyme (DM) cells attached to the basement membrane of IEE. ( $\mathrm{g}-\mathrm{i}$ ) At E60, the DM3 germ developed into late bell (secretory stage), with parts of the IEE and dental papilla cells at the cusp tip differentiated into ameloblasts and odontoblasts (O). Enamel $(E)$ and pre-dentin (pD)

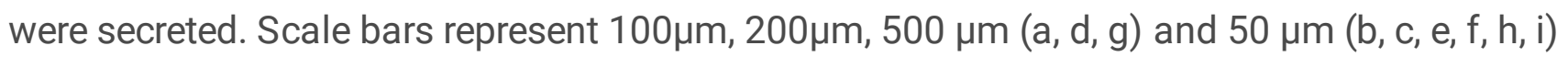

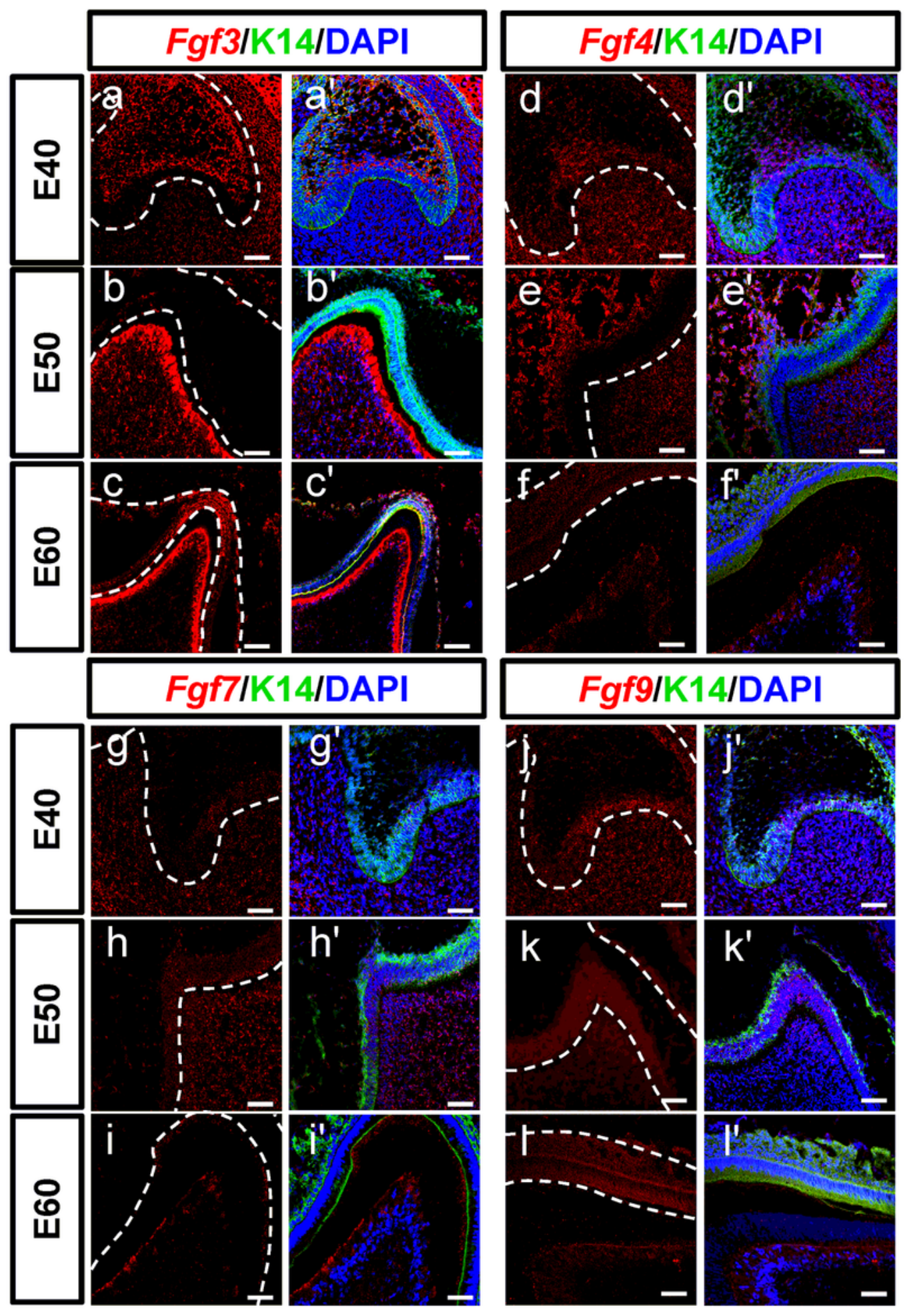

Figure 2 
Dynamic expression of genes encoding FGF ligands during morphogenesis of DM3. (a-I, a'-l') Fluorescence in situ hybridization (FISH) showing the expression of genes (red) with immunofluorescence (IF) for Cytokeratin14 (K14) protein (green) to clarify the epithelium, and nuclei were stained with DAPI (blue) from the cap to late bell stages. (a-l) only these genes are represented (red); (a-c) Fgf3 expression from the cap to late bell stages; (d-f) Fgf4 expression from the cap to secretory stages; (g-i) Fgf7 and (j-I) Fgf9 expression from the cap to late bell stages. scale bar $50 \mu \mathrm{m}$

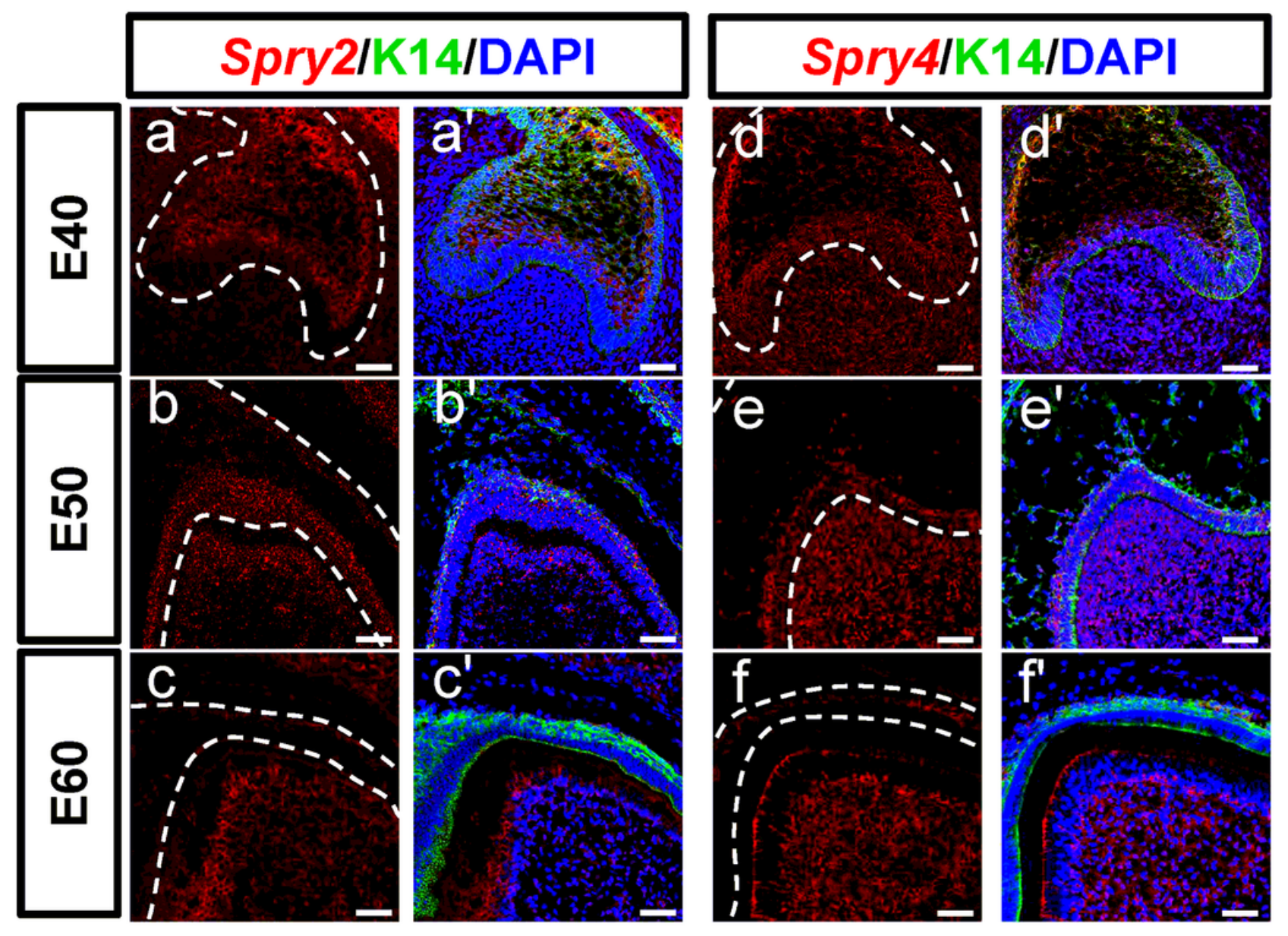

Figure 3

Dynamic expression of genes encoding FGF inhibitors during morphogenesis of DM3. FISH (red) with K14 protein (green) as well as nuclei were stained with DAPI (blue) from the cap to secretory stages (a-f, $\left.a^{\prime}-f^{\prime}\right)$. (a-f) only these genes represented (red). (a-c) Spry2 expression from the cap to secretory stages; (df) Spry4 expression from the cap to secretory stages. scale bar $50 \mu \mathrm{m}$ 


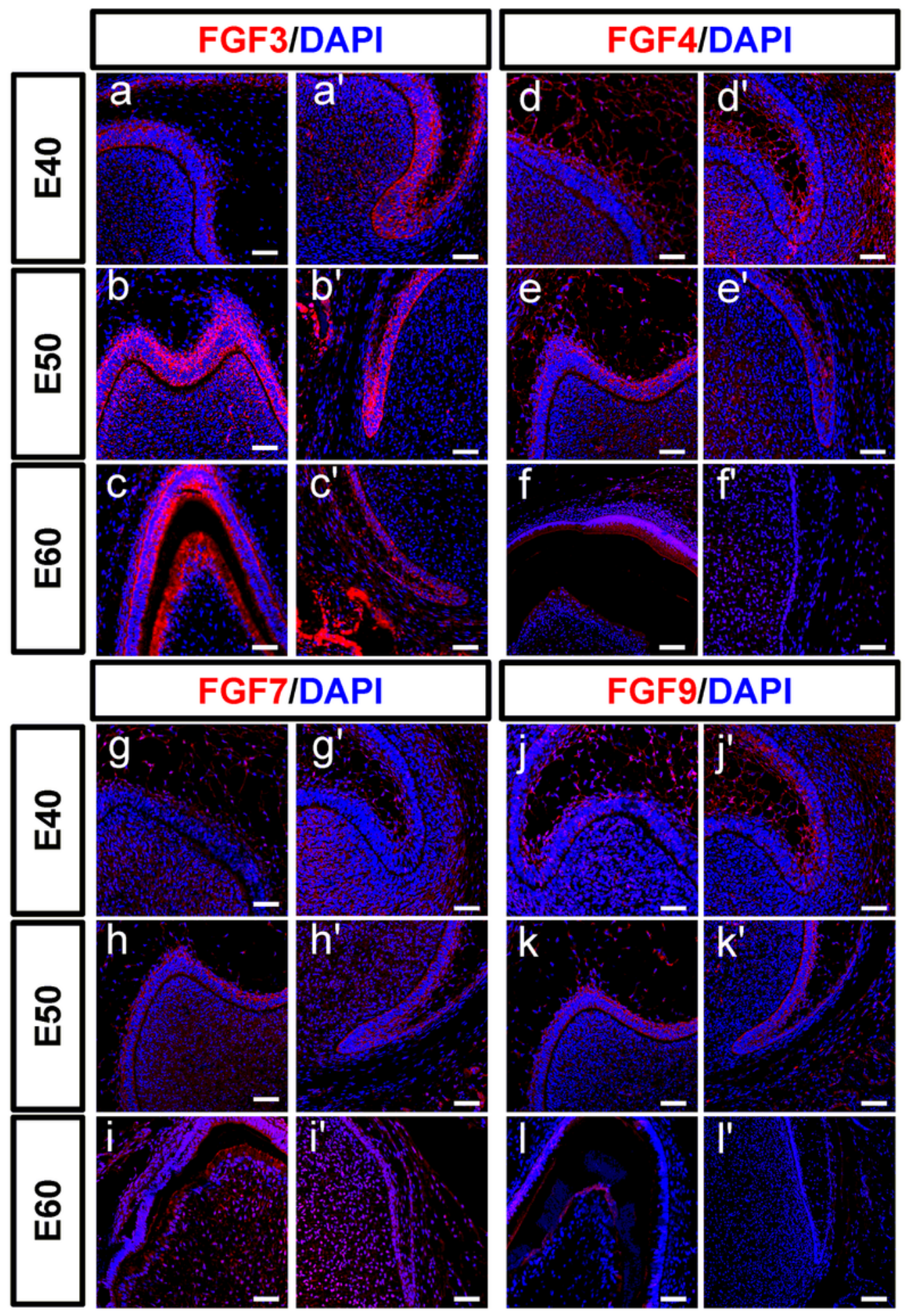

Figure 4

Distribution of FGF ligands during morphogenesis of DM3. (a-I, a'- $\left.I^{\prime}\right)$ Detection of FGF proteins expression by IF from the cap to secretory stages. (a-c, a'-c') FGF3 expression from the cap to secretory stages. (d-f, $\left.d^{\prime}-f^{\prime}\right)$ FGF4 expression from the cap to secretory stages. ( $\left.g-i, g^{\prime}-i^{\prime}\right)$ FGF7 and $\left(j-1, j^{\prime}-I^{\prime}\right)$ FGF9 expression from the cap to secretory stages. scale bar $50 \mu \mathrm{m}$ 


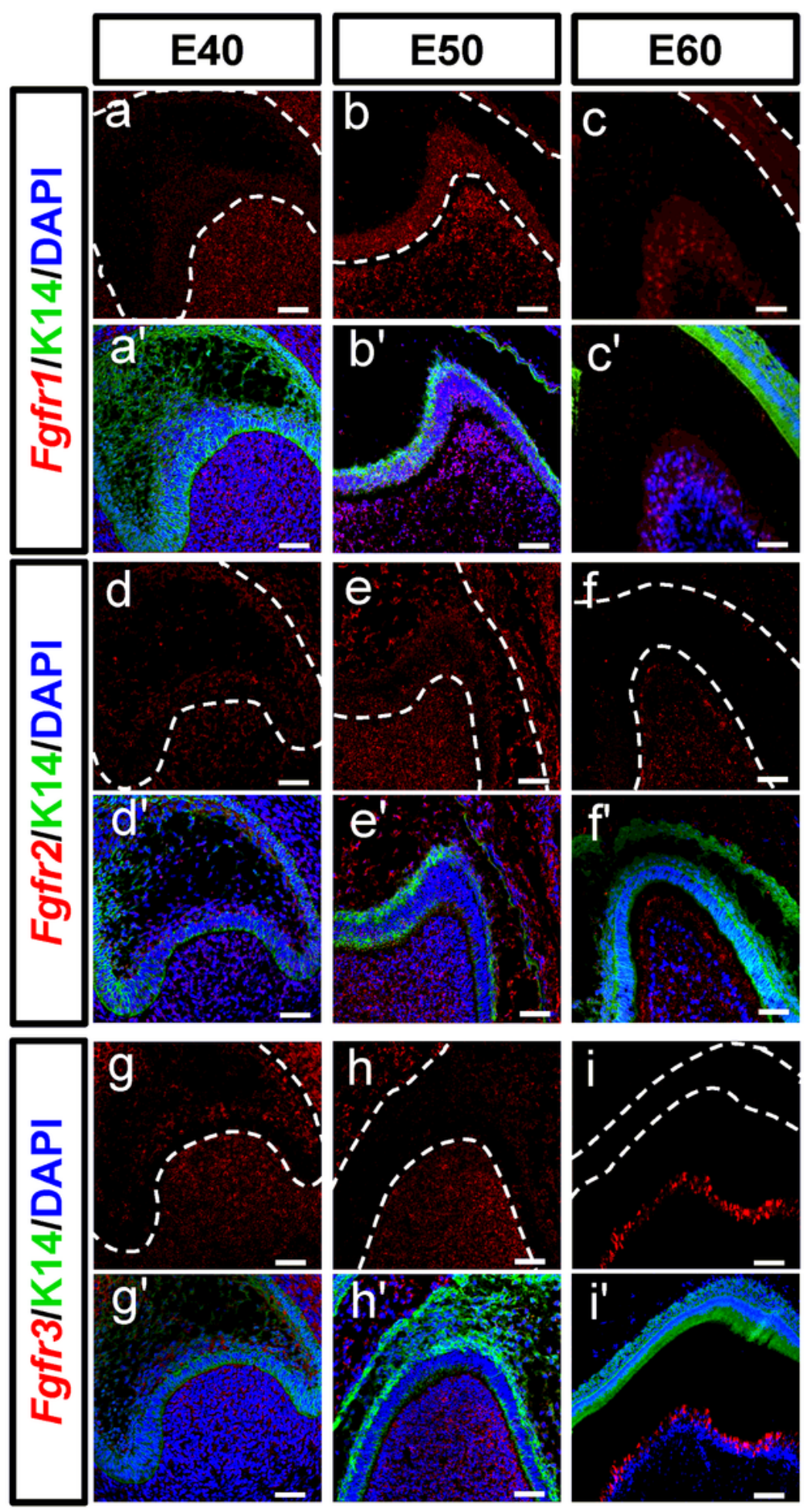

Figure 5

Dynamic expression of genes encoding FGF receptors during morphogenesis of DM3. FISH (red) with K14 protein (green) as well as nuclei were stained with DAPI (blue) from the cap to secretory stages (a-i, $\left.a^{\prime}-i^{\prime}\right)$. (a-i) only these genes represented (red). (a-c) Fgfr1 expression from the cap to secretory stages. (d-f) Fgfr2 expression from the cap to secretory stages, (g-i) Fgfr3 expression from the cap to secretory stages detected as mentioned above. scale bar $50 \mu \mathrm{m}$ 

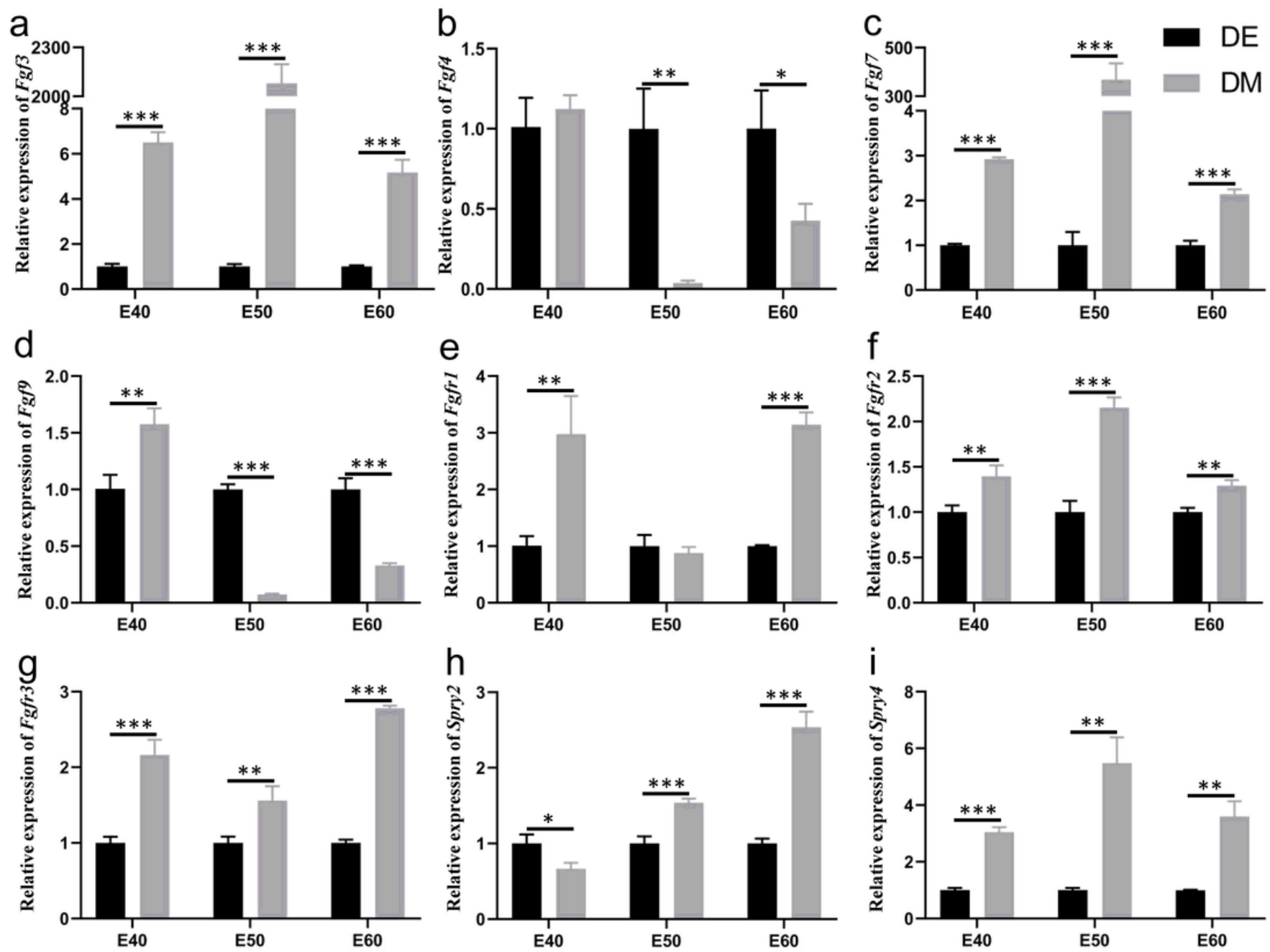

Figure 6

Quantitative gene expression dynamics related to the FGF pathway and its inhibitors of DM3. (a-d) Expression levels of Fgf3, Fgf4, Fgf7, and Fgf9 from the cap to secretory stages. (e-g) Expression levels of Fgfr1, Fgfr2, and Fgfr3 from the cap to secretory stages. (h-i) Expression levels of Spry2 and Spry4 from the cap to secretory stages. Significance was indicated by asterisks $\left(* P<0.05,{ }^{\star *} P<0.01\right.$, or $* \star \star P<$ $0.001)$. 


\section{E40}

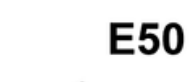

Fgf3
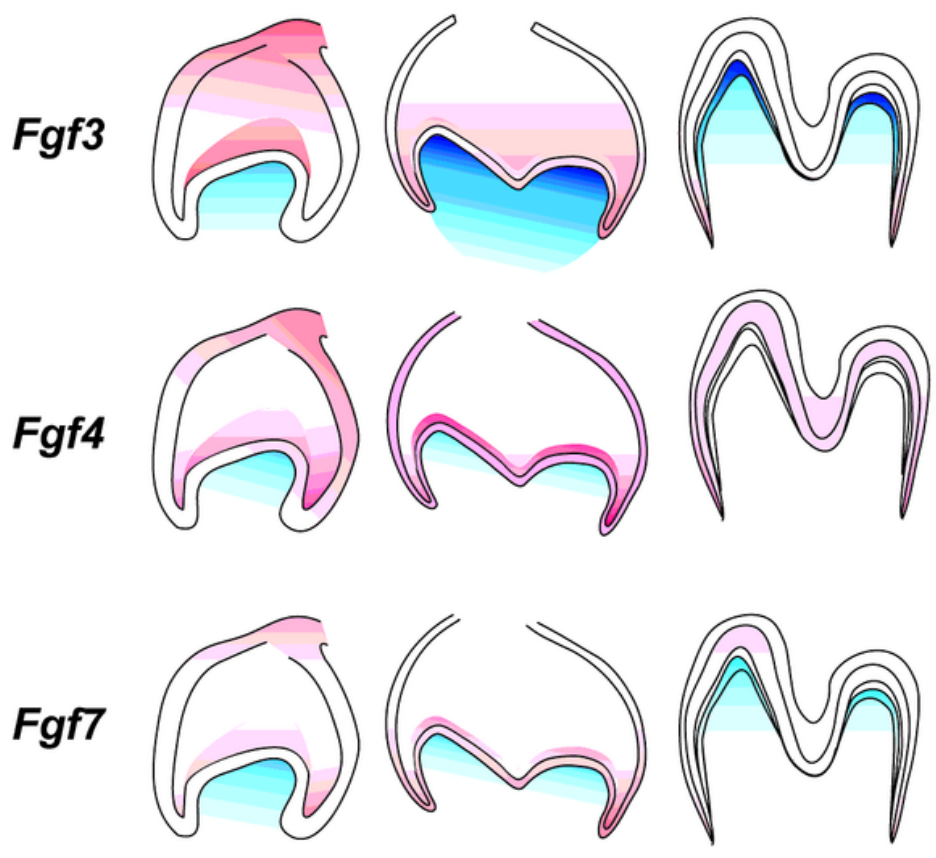

Fgf9
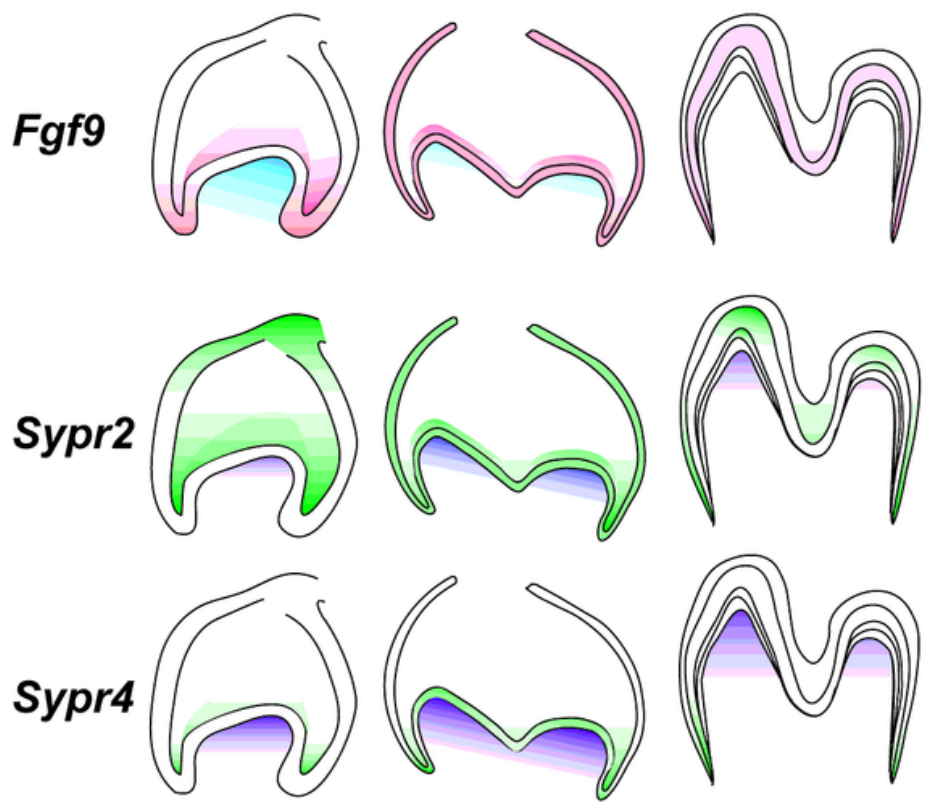

E40
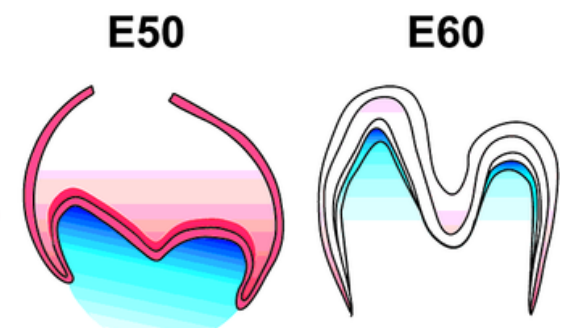

Fgfr2
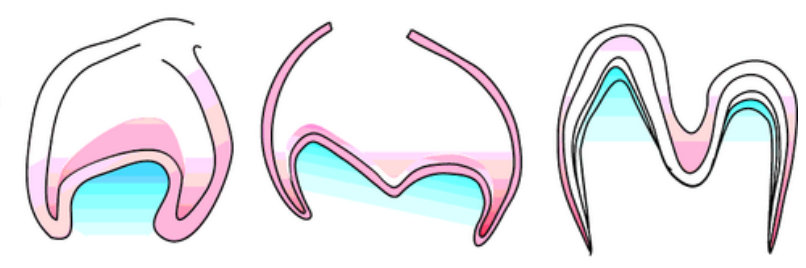

Fgfr3
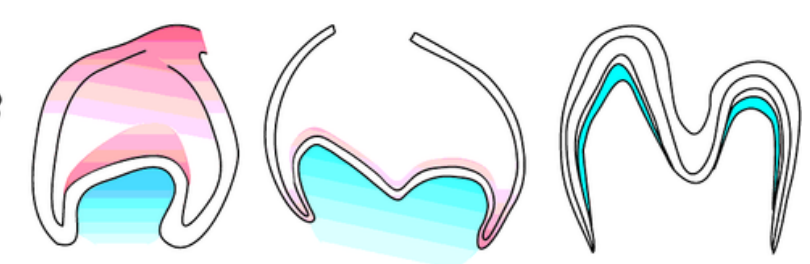

$F g f$
$D E$

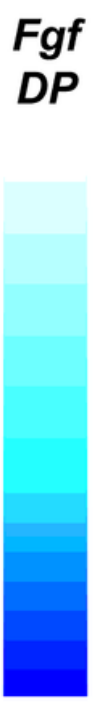

Figure 7

Pattern diagram of gene expression dynamics of the FGF pathway and its inhibitors of DM3. Lighter color means less expression, darker color means higher relative expression. (DM: dental mesenchyme, DE: dental epithelium)

\section{Supplementary Files}

This is a list of supplementary files associated with this preprint. Click to download.

- Supplementaltable.docx 
- SupplementaryFigure1.tif

- SupplementaryFigure2.tif

- SupplementaryFigure3.tif

- SupplementaryFigure4.tif 